\section{Specific binding of the transcription factor sigma-54 to promoter DNA}

\section{Martin Buck \& Wendy Cannon}

AFRC Nitrogen Fixation Laboratory, University of Sussex, Brighton BN1 9RQ, UK

A CENTRAL event in transcription is the assembly on DNA of specific complexes near the initiation sites for RNA synthesis. Activation of transcription by one class of enhancer-binding proteins requires an RNA polymerase holoenzyme ${ }^{1}$ containing the specialized transcription factor, sigma-54 $\left(\sigma^{54}\right)$. We report here that $\sigma^{54}$ alone specifically binds to promoter DNA and is responsible for many of the close contacts between RNA polymerase holoenzyme and promoter DNA, a property proposed for the major $\sigma^{70}$ protein family. Binding of $\sigma^{54}$ to promoter DNA is not equivalent to that of holoenzyme suggesting that there is a constraint on $\sigma^{54}$ conformation when bound with core RNA polymerase. Footprints indicate $\sigma^{54}$ is at the leading edge of DNAbound holoenzyme. Like the holoenzyme ${ }^{1-4}, \sigma^{54}$-binding to promoter DNA does not result in DNA strand separation. Instead the specific DNA-binding activity of $\sigma^{54}$ assists assembly of a closed promoter complex. This complex can be isomerized to the open (DNA melted) complex by activator protein ${ }^{5,6}$, but promoterbound $\sigma^{54}$ alone cannot be induced to melt DNA. The pathway leading to productive transcription is similar to that proposed for eukaryotic RNA polymerase II systems.

The promoter sequence recognized by the holoenzyme $\left(\mathrm{E} \sigma^{54}\right)$ is generally characterized by the presence of GG and GC doublets 24 and 12 base pairs, respectively, upstream of the transcription initiation point (Table 1 ; refs 1,3 ), and activation requires binding of the appropriate activator protein upstream of $E \sigma^{54}$ at enhancer sequences ${ }^{7-9}$. Using conditions for detecting closed promoter complexes at the $\sigma^{54}$-dependent Klebsiella pneumoniae nitrogen fixation (nif) promoters ${ }^{10}$ we footprinted the nifH promoter and a mutant variant, nifH049 (Table 1) which forms a stronger closed complex ${ }^{3,11}$. We were able to detect $\mathrm{E} \sigma^{54}$ complexes bound at the nifH and nif $\mathrm{HO} 49$ promoters, and $\sigma^{54}$ bound at the nif $\mathrm{HO49}$ promoter (Figs $1 a$ and 3 ). The nif $H$ promoter did not detectably bind $\sigma^{54}$ (Fig. 3, lanes $16,17)$. The complexes with $\mathrm{E} \sigma^{54}$ and the nifH and nifH049 promoters were not equivalent. The ExoIII digestion block was 3-4 base pairs (bp) closer to the transcription initiation site in the nif $\mathrm{HO} 49$ promoter. Thus $\mathrm{E} \sigma^{54}$ seemed to establish more contacts at the nif $\mathrm{HO} 49$ promoter. In the absence of core RNA polymerase subunits, $\sigma^{54}$ bound to the nifH049 promoter to produce a clear ExoIII block (Fig. $1 a$ ), but only partially protected conserved guanine residues at $-24,-25$ and -13 from dimethylsulphate attack (Fig. $1 b$ ). The $\sigma^{54}$-dependent ExoIII block at the nif $H 049$ promoter was 3-4 bp closer to the transcription initiation site than when $\mathrm{E} \sigma^{54}$ bound (Fig. 1a), indicating $\sigma^{54}$ is conformationally constrained by core polymerase. Unlike $\mathrm{E} \sigma^{54}$ (ref. 11), binding of $\sigma^{54}$ did not increase reactivity of the $\mathrm{T}$ at -9 towards $\mathrm{KMnO}_{4}$ (data not shown). Thus $\sigma^{54}$ did not seem to distort the DNA. DNase 1 footprints revealed that the DNA was covered to a similar extent by $\sigma^{54}$ and $\mathrm{E} \sigma^{54}$, but that the major $\mathrm{E} \sigma^{54}$ footprint extended about 7 bases further upstream than did the $\sigma^{54}$ footprint (Fig. 1c). Gel mobility shift assays also demonstrated that the complex forming with $\sigma^{54}$ was different to that forming in the presence of $\mathrm{E}^{54}$, the latter complex having a much reduced mobility (data not shown).

To extend our analysis of $\sigma^{54}$ binding to include a naturally occurring promoter sequence (Table 1 ) we examined the binding of $\mathrm{E} \sigma^{54}$ (ref. 3) and $\sigma^{54}$ to the Rhizobium meliloti nifH promoter (Fig. 2). Both $\sigma^{54}$ and $\mathrm{E} \sigma^{54}$ produced clear blocks to ExoIII digestion, with the $\sigma^{54}$ block being about 4 bp closer to the transcription initiation site than the $\mathrm{E} \sigma^{54}$ block. Dimethylsulphate and DNase 1 footprints again revealed that $\sigma^{54}$ was responsible for most of the close contacts detected between holoenzyme and the $R$. meliloti nif $H$ promoter (Gs at $-14,-25$, -26 ; Fig. 2). Holoenzyme covered $\sim 32$ bp and $\sigma^{54}$ covered 27 bp of promoter DNA as judged by DNase 1 footprinting (Fig. 2). Footprints on the bottom strand showed a single Exolli block for $\sigma^{54}$ and $E \sigma^{54}$ at around -34 , protection of Gs $-13,-22$ and -24 by $\sigma^{54}$ and $E \sigma^{54}$ and a DNase 1 footprint from -34 to -5 for $\sigma^{54}$ and $\mathrm{E} \sigma^{54}$.

The nif $H 049$ and $R$. meliloti nifH promoters have a ' $T$ '-tract from -14 to -17 that distinguishes them from the nif $H$ promoter $^{11}$ and is necessary for the binding of $\sigma^{54}$ (Table 1). $R$. meliloti and nifH049 templates in which dT was replaced by $\mathrm{dU}$, effectively removing the 5-methyl group of $\mathrm{T}$, bound $\mathrm{E} \sigma^{54}$ but not $\sigma^{54}$ (Fig. 3; Table 1). In complementary experiments we constructed wild-type sequence $K$. pneumoniae nif $H$ templates in which $\mathrm{dC}$ was replaced by $\mathrm{dm}^{5} \mathrm{C}$ and found that $\sigma^{54}$ then bound (Fig. 3 ). The sequence from -14 to -17 is therefore critical for $\sigma^{54}$ binding. Specifically the methyl groups in the DNA major groove are important (Table 1).

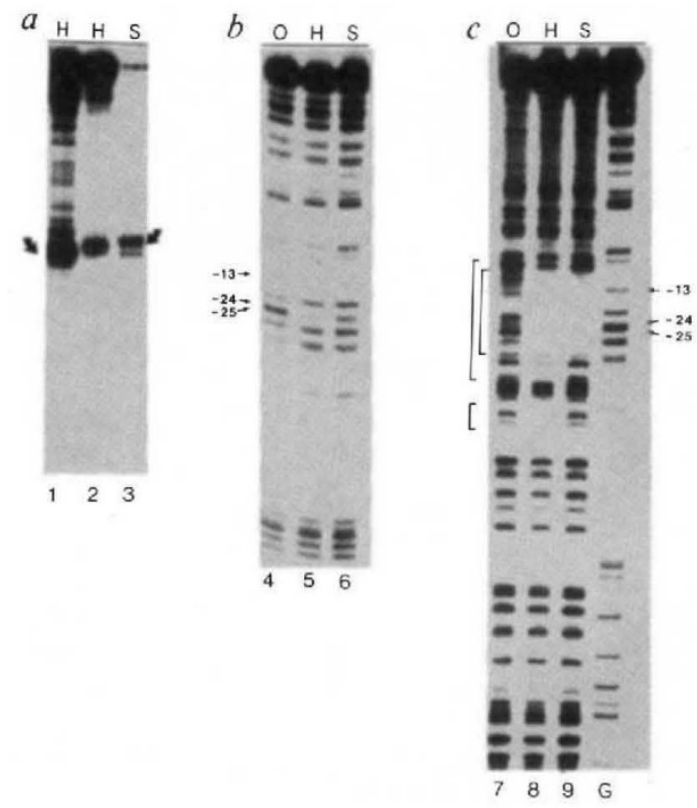

FIG. 1 Footprints demonstrating the binding of $E \sigma^{54}$ and $\sigma^{54}$ to the $K$. pneumoniae nifH and nifHO49 promoters. a, Exonuclease III footprints; $b$, dimethylsulphate footprints; $c$, DNase 1 footprints, H, Holoenzyme; S, $\sigma^{54}$; $\mathrm{O}$, no addition. Arrows mark Exolll blocks due to $\mathrm{E} \sigma^{54}$ or $\sigma^{54}$ binding; brackets, the extent of the DNase 1 footprint. Lane 1, wild-type nifH promoter; lanes 2-9, nifH049 promoter. Lane G, Chemical cleavage of DNA at G residues. $\mathrm{E} \sigma^{54}$ bound the nifH and nifHO49 promoters, $\sigma^{54}$ bound the nif $H 049$ promoter. Footprints were conducted as described previously ${ }^{10}$ in $25 \mathrm{mM}$ Tris-acetate, $8 \mathrm{mM}$ magnesium acetate, $10 \mathrm{mM} \mathrm{KCl}, 1 \mathrm{mM}$ dithiothrietol, $3.5 \%(\mathrm{w} / \mathrm{v})$ polyethylene glycol, pH 8.0. Template DNA (2.4 nM) was incubated with $125 \mathrm{nM}$ core RNA polymerase $(\mathrm{E})$ and $280 \mathrm{nM} \sigma^{54}\left(\mathrm{E} \sigma^{54}\right)\left(\mathrm{E}\right.$ and $\sigma^{54}$ were previously combined and warmed at $30^{\circ} \mathrm{C}$ for $5 \mathrm{~min}$ before addition) at $30^{\circ} \mathrm{C}$ in a $50-\mu$ l assay. When $\sigma^{54}(560 \mathrm{nM})$ alone was used, this was also preincubated before adding to template DNA. Binding was for $20 \mathrm{~min}$ before addition of footprinting reagent. In DNase 1 footprints, salmon sperm DNA $\left(270 \mu \mathrm{g} \mathrm{ml}^{-1}\right)$ was included in the incubations. K. pneumoniae $\sigma^{54}$ was free of $\beta$ and $\beta^{\prime}$ RNA polymerase subunits as judged by stained, overloaded gels. Equivalent exonuclease III and DMS footprints were obtained when the binding buffer was supplemented with $100 \mathrm{mM} \mathrm{KCl}$ or footprints were conducted in $50 \mathrm{mM}$ Tris-acetate, $100 \mathrm{mM}$ potassium acetate, $8 \mathrm{mM}$ magnesium acetate, $27 \mathrm{mM}$ ammonium acetate, $1 \mathrm{mM}$ dithiothrietol, $3.5 \%(\mathrm{w} / \mathrm{v})$ polyethylene glycol ${ }^{2}$. Thus binding of $\sigma^{54}$ persists at physiologically relevant salt concentrations. Positions of digestion blocks were estimated from $\mathrm{G}$ and $\mathrm{C}+\mathrm{T}$ chemical cleavage sequencing ladders. 

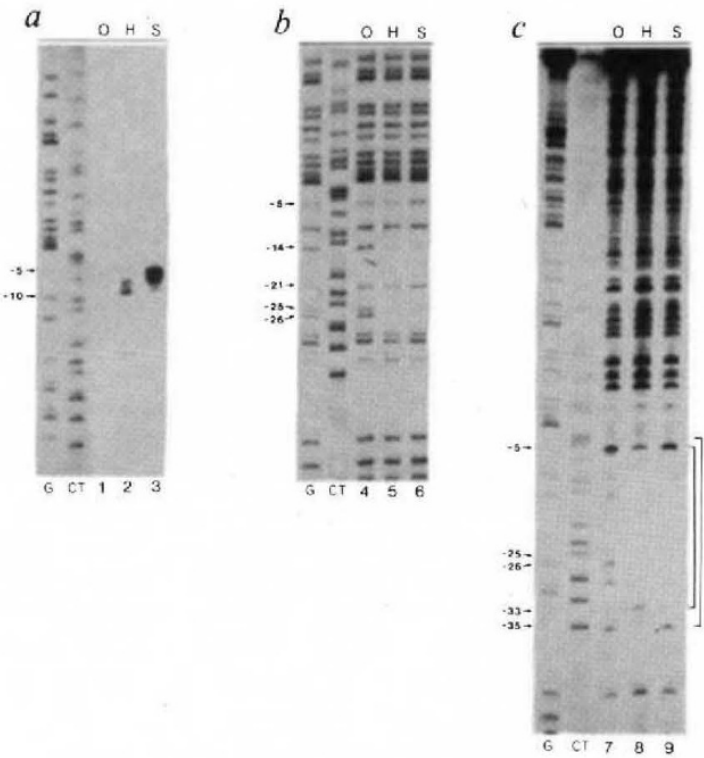

FIG. 2 Footprints demonstrating the binding of $\mathrm{E} \sigma^{54}(\mathrm{H})$ and $\sigma^{54}(\mathrm{~S})$ to the $R$. meliloti nifH promoter. $a$, Exonuclease III footprints; $b$, dimethylsulphate footprints; $c$, DNase 1 footprints. Conserved Gs at $-14,-25,-26$ were protected from dimethylsulphate attack by $\sigma^{54}$ and $\mathrm{E}^{54}$. Assays were as for Fig. 1, and footprints persisted in buffer supplemented with $100 \mathrm{mM}$ $\mathrm{KCl}$ or when the $100 \mathrm{mM}$ potassium acetate-containing buffer was used. The $\sigma^{54}$ Exoll block persisted down to $15 \mathrm{nM} \sigma^{54}$, and shifting the $\sigma^{54}$ block to that characteristic of $E \sigma^{54}$ needed a minimum of $2 \mathrm{nME}$ at $280 \mathrm{nM}$ $\sigma^{54}$. Serial dilution of $\mathrm{E} \sigma^{54}$ resulted in a gradual loss of the $\mathrm{E} \sigma^{54}$ ExollI block without the appearance of the block characteristic of $\sigma^{54}$ alone.

The closed complex that forms at the nifH049 promoter can be isomerized to the open complex by the NtrC S160F enhancerbinding activator protein ${ }^{11-13}$. But when prebound $\sigma^{54}$ at nif $\mathrm{H} 049$ was incubated with $\mathrm{NtrC}$ S160F protein no evidence for DNA melting (using DNA reactivity towards $\mathrm{KMnO}_{4}$ as an index of denaturation) was obtained (data not shown). Thus $\sigma^{54}$ seems unable to function in the process of open complex formation unless bound as holoenzyme.

In summary, $\sigma^{54}$ functions through assisting assembly of a preinitiation complex consisting of $\sigma^{54}$-holoenzyme and promoter DNA at a site centred about $18 \mathrm{bp}$ upstream of the
TABLE 1 Promoter sequences and template requirements for binding $\sigma^{54}$

\begin{tabular}{|c|c|c|}
\hline Promoters & & $\sigma^{54}$ Binding \\
\hline Wild-type nifH & CTG்ĠTATGTTCCCTG்ĊA & - \\
\hline $\begin{array}{l}\text { nif } H 049 \\
R \text { meliloti nifH }\end{array}$ & 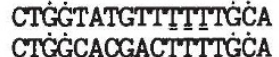 & $\begin{array}{l}+ \\
+\end{array}$ \\
\hline
\end{tabular}

\begin{tabular}{lccc} 
& & \multicolumn{2}{c}{ Footprints } \\
& Template sequences & $\mathrm{E} \sigma^{54}$ & $\sigma^{54}$ \\
nifH & CCCTGCA & +++ & - \\
nifHO49 & TTTTGCA & +++ & +++ \\
RmH & TTTTGCA & +++ & +++ \\
nifHO49-dU & UUUUGCA & +++ & - \\
RmH-dU & UUUUGCA & +++ & - \\
nifH- $\mathrm{dm}^{5} \mathrm{C}$ & $\mathrm{m}^{5} \mathrm{Cm}^{5} \mathrm{Cm}^{5} \mathrm{CTGm} \mathrm{CA}^{5}$ & +++ & + \\
\hline
\end{tabular}

Binding sites for $\mathrm{E} \sigma^{54}$ are characterized by $\mathrm{GG}$ and $\mathrm{GC}$ doublets separated by $10 \mathrm{bp}$ and centred roughly $18 \mathrm{bp}$ upstream of the transcription initiation site. A T-tract upstream of the GC doublet is important for the binding of $\mathrm{E} \sigma^{54}$ and $\sigma^{54}$ (refs 3,22 ). The nifHO49 and $R$. meliloti nifH promoters bound $\sigma^{54}$ but binding to the wild-type nifH promoter was not detected (Figs 1-3). When templates were prepared in which the 5-methyl group of T was absent (dU), $\sigma^{54}$ did not detectably bind (Fig. 3). Introduction of 5 -methyl $\left(\mathrm{dm}^{5} \mathrm{C}\right)$ into templates increased binding of $\sigma^{54}$ (Fig. 3).

transcription initiation point. A predominant event in binding holoenzyme to promoter DNA is recognition of DNA by $\sigma^{54}$. The nonequivalence in the $\sigma^{54}$ recognition sequence when the -25 and -13 elements are compared presumably enables the orientation of DNA-bound $\sigma^{54}$ to define the direction of transcription.

Detection of $\mathrm{E} \sigma^{54}$ binding when $\sigma^{54}$ binding is not evident indicates core RNA polymerase reduces the promoter dissociation rate of $\sigma^{54}$. It is possible that the binding specificities of $\sigma^{54}$ and $\mathrm{E} \sigma^{54}$ are different, suggesting a source of favourable binding energy for the holoenzyme. Notably $\sigma^{54}$ functions with the same core RNA polymerase subunits as does the major eubacterial sigma factor, $\sigma^{70}$, but seems to associate (at least in the absence of DNA template) relatively weakly with core RNA polymerase ${ }^{14}$. It is possible that $\sigma^{54}$ can bind the template and then associate with core RNA polymerase, possibly in contrast to $\sigma^{70}$ which seems to bind only as holoenzyme ${ }^{15}$. It seems that $\sigma^{54}$ is at the leading edge of the holoenzyme, positioned to extend over DNA to be melted during open complex formation, implying $\sigma^{54}$ might directly participate in DNA melting. Open promoter complex formation occurs efficiently in response to

FIG. 3 Binding of $E \sigma^{54}(\mathrm{H})$ and $\sigma^{54}(\mathrm{~S})$ to templates altered by the replacement of $d T$ by $\mathrm{dU}$ or of $\mathrm{dC}$ by $\mathrm{dm}^{5} \mathrm{C}$ (see also Table 1). Binding was assayed by exonuclease III footprinting and blocks are marked with arrows. Lanes 1-3, nifHO49 dT template footprints; lanes 4-6, nif $H 049$ dU template footprints; lanes $7-9, R$. meliloti nif $H$ dU template footprints; lane 10, R. meliloti nifH dT template footprint. Wild-type K. pneumoniae nifH $\mathrm{dm}^{5} \mathrm{C}$ template footprints, lanes 11-15. For comparison, footprints of the wild-type K. pneumoniae dT template are shown in lanes 16 and 17 . Assays for lanes 15 and 17 contained $1.2 \mu \mathrm{M} \sigma^{54}$. Lanes 16 and 17 were twofold overloaded. Lane G, chemical cleavage of DNA at G residues. Templates were generated by polymerization using nucleotide triphosphate mixes containing either dUTP or $\mathrm{dm}^{5} \mathrm{CTP}$ rather than dTTP or dCTP, and thus the top nontemplate strand only is modified (the strand visualized in the
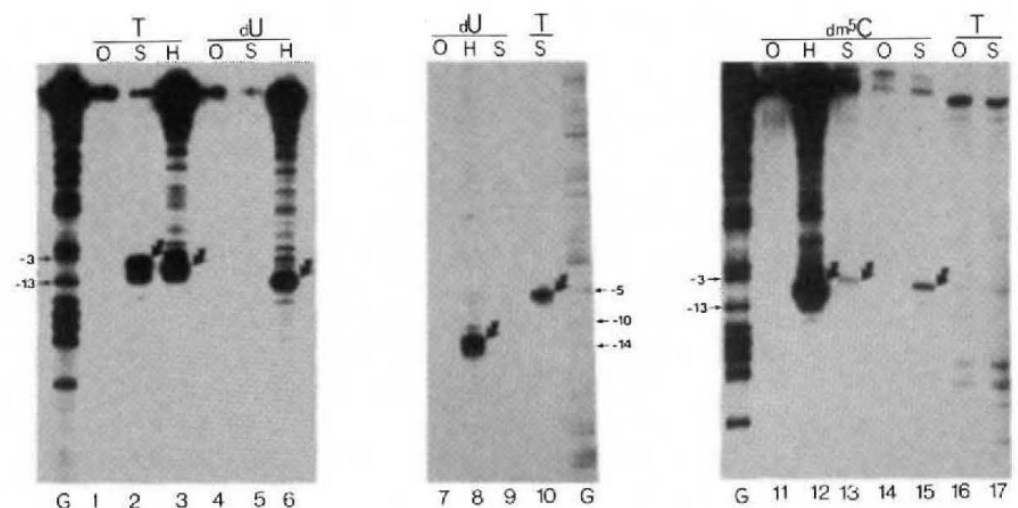
footprints). Substituting $d T$ by $d U$ effectively removes the $C^{5}$-methyl group of $d T$ found in the major groove of DNA, and replacement of $d C$ by $\mathrm{dm}^{5} \mathrm{C}$ effectively introduces $C^{5}$-methyl. No binding of $\sigma^{54}$ to the wild-type nifH dT template was detected (lane 17 and unpublished data). Replacement of dT by $\mathrm{dU}$ greatly reduced $\sigma^{54}$ binding (lanes 5,9 ). Replacement of $\mathrm{dC}$ by $\mathrm{dm}^{5} \mathrm{C}$ increased $\sigma^{54}$ binding (lanes 13,15). Differences in DNA geometries may also contribute to the preferential binding of $\sigma^{54}$ to dT rather than $\mathrm{dm}^{5} \mathrm{C}$ containing templates. As anticipated from the conservation of $\mathrm{G} \cdot \mathrm{C}$ base

pairs at $-24,-25$ and -13 and their protection from methylation by $\mathrm{E}^{54}$ and $\sigma^{54}$ (Fig. 2; refs 2,10 ) replacement of $d G$ by $C^{7}$-deaza guanine resulted in templates that failed to bind $E \sigma^{54}$ or $\sigma^{54}$ as judged by DNase 1 footprinting (data not shown). The sequence TTCGCI rather than TIIGCA from -16 to -11 in the enteric ginAp2 promoters may explain why the binding of $\sigma^{54}$ is not readily detected at this sequence ${ }^{19,20}$. Proposed domain structures for $\sigma^{54}$ indicate that DNA recognition may involve a helix-turn-helix structure ${ }^{18,21}$ 
the activator only when $\sigma^{54}$ is bound as holoenzyme at the promoter. The conformation of $\sigma^{54}$ in the holenzyme, or the RNA polymerase subunits themselves, may also be important for DNA melting. That $\sigma^{54}$ binds to promoter DNA but does not melt it is consistent with all known $\sigma^{54}$-dependent transcription systems that have a strict requirement for an activator protein ${ }^{1}$. Separation of promoter recognition and DNA melting functions permits $\mathrm{E} \sigma^{54}$ to respond to many stimuli of gene expression through their influences on the activity of the activator protein. Activator dependence and assembly of the preinitiation complex in its absence strongly suggests a broad similarity to eukaryotic RNA polymerase II transcription ${ }^{16,17}$.

Received 24 March; accepted 10 June 1992

1. Kustu, S., Santero, E., Keener, J., Popham. D. \& Weiss, D. Microbiol. Rev. 53, 367-379 (1989)

2. Popham, D. L., Szeto, D., Keener, J. \& Kustu, S. Science 243, 629-635 (1989).

3. Morett, E. \& Buck, M. J. molec. Biol. 210, 65-77 (1989).

4. Sasse-Dwight, S. \& Gralla, J. D. Proc. natn. Acad. Sci. U.S. A. 85, 8934-8938 (1988).

5. Weiss. D. S., Batut, J., Klose, K. E., Keener, J. \& Kustu, S. Cell 67, 155-167 (1991).

6. Austin, S. \& Dixon, R. EMBO J. 6, 2219-2228 (1992).

7. Buck, M. Miller, S. Drummond M. \& Dixon, R. Nature 320, 374-378 (1986)

8. Reitzer, L. J. \& Magasanik, B. Cell 45, 785-792 (1986)

9. Morett, E. \& Buck, M. Proc. natn. Acad. Sci. U.S.A. 85, $9401-9405$ (1988).

10. Buck, M. \& Cannon. W. Molec. Microbiol. (in the press).

11. Cannon, W. \& Buck, M. J. molec. Biol. 225, 271-286 (1992).

12. Popham, D., Kenner, J. \& Kustu, S. J. biol. Chem. 266, 19510-19518 (1991)

13. Austin, S. Kundrot, C. \& Dixon, R. Nucleic Acids Res. 19, 2281-2287 (1991).

14. Lesley, S. A. Brow, M. A. D. \& Burgess, R. L biol Chem 266 2632-2638 (1991)

15. Helman, J. D. \& Chamberlain, M. J. A. Rev. Biochem. 57, 839-872 (1988).

16. Greenblatt, J. Cell 66, 1067-1070 (1991).

7. Wang, W., Carey, M. \& Gralla, J. D. Science 255, 450-453 (1992).

18. Sasse-Dwight, S. \& Gralla, J. D. Cell 62, 945-954 (1990)

19. Kustu, S., Sei, K. \& Keener, J. in Regulation of Gene Expression, Symp. Soc. gen. Microbiol. (eds Booth, I. \& Higgins, C.) 139-154 (Cambridge Univ. Press, 1986).

20. Ninfa, A. J. Reitzer, L. J. \& Magasanik, B. Cell 50, 1039-1046 (1987)

21. Coppard, J. R. \& Merrick, M. J. Molec. Microbiol. 5, 1309-1317 (1991)

22. Claverie-Martin, F. Magasanik, B. Proc. natn. Acad. Sci. U.S.A. 88, 1631-1635 (1991)

ACKNOWLEDGEMENTS. We thank S. Austin for purified $K$. pneumoniae proteins; M. Moore for discussions; S. Austin, R. Dixon, M. Drummond, M. Merrick and B. Smith for comments on the manuscript and $\mathrm{R}$. Foote for typing.

\section{Different $\beta$-subunits determine G-protein interaction with transmembrane receptors}

\section{Kleuss*, H. Scherüb $\dagger$, J. Hescheler†, G. Schultz† \& B. Wittig*}

* Institut für Molekularbiologie und Biochemie, and

† Institut für Pharmakologie, Freie Universität Berlin, Arnimallee 22,

D-1000 Berlin 33, Germany

REGULATORY GTP-binding proteins (G proteins) are membraneattached heterotrimers $(\alpha, \beta, \gamma)$ that mediate cellular responses to a wide variety of extracellular stimuli ${ }^{1,2}$. They undergo a cycle of guanine-nucleotide exchange and GTP hydrolysis, during which they dissociate into $\alpha$-subunit and $\beta \gamma$ complex ${ }^{1}$. The roles of $G$-protein $\alpha$-subunits in these processes and for the specificity of signal transduction are largely established; the $\beta$ - and $\boldsymbol{\gamma}$-subunits are essential for receptor-induced G-protein activation and seem to be less diverse and less specific. Although the complementary DNAs for several $\beta$-subunits have been cloned ${ }^{2,5-8}$, isolated subunits have only been studied as $\beta \gamma$ complexes ${ }^{3,9-12}$. Functional differences have been ascribed to the $\gamma$-subunit on the basis of extensive sequence similarity among $\beta$-subunits and apparent heterogeneity in $\gamma$-subunit sequences ${ }^{13,14}$. $\beta \gamma$ complexes can interact directly or indirectly with different effectors ${ }^{10,11,15-20}$. They seem to be interchangeable in their interaction with pertussis toxin-sensitive $\alpha$-subunits ${ }^{3}$, so we tested this by microinjecting antisense oligonucleotides into nuclei of a rat pituitary cell line to suppress the synthesis of individual $\beta$-subunits selectively. Here we show that two out of four subtypes of $\beta$-subunits tested $\left(\beta_{1}\right.$ and $\beta_{3}$ ) are selectively involved in the signal transduction cascades from muscarinic $M_{4}$ (ref. 4) and somatostatin receptors, respectively, to voltage-dependent $\mathrm{Ca}^{2+}$ channels.

We have established nuclear microinjection of short selective antisense oligonucleotides as a general method to study G protein function in intact cells ${ }^{21}$. The effects of 'knocking out' the expression of individual G-protein $\alpha$-subunits are measured in a single cell either electrophysiologically or by immunofluorescent labelling of the targeted proteins. Here we apply this approach to elucidate the role of $\beta$-subunit subtypes in the differential coupling of $G$ proteins to the same receptor/G protein/effector systems in $\mathrm{GH}_{3}$ cells, a rat pituitary cell line.

Cells that have been injected with the antisense oligonucleotide $\beta$-com (see Fig. 1 legend), which targets messenger RNA of all known four $\beta$-polypeptides, contain reduced amounts of immunostainable $\beta$-subunits (data not shown). The disappearance of $\beta$-subunits parallels the functional loss of inhibitory hormonal effects on $\mathrm{Ca}^{2+}$ currents. $\mathrm{Ca}^{2+}$ currents in $\mathrm{GH}_{3}$ cells are probably due to the activation of L-type channels and are inhibited to $70-80 \%$ of control currents by carbachol and somatostatin $^{21}$. One day after injection of the oligonucleotide $\beta$-com, neither hormone reduced the $\mathrm{Ca}^{2+}$ current (Fig. 1); this effect lasted for one more day. The original hormone sensitivity of the $\mathrm{Ca}^{2+}$ channel was restored by $\sim 60 \mathrm{~h}$ after injection.

To study the question of preferential interaction between the activated receptor and $\mathrm{G}_{\mathrm{O}}$ proteins containing a given $\beta$ subtype, $\mathrm{GH}_{3}$ cells were injected with antisense oligonucleotides that can specifically hybridize with the mRNA of one particular $\beta$-subtype (Figs 2 and 3). Neither $\beta_{2}$ - nor $\beta_{4}$-specific antisense

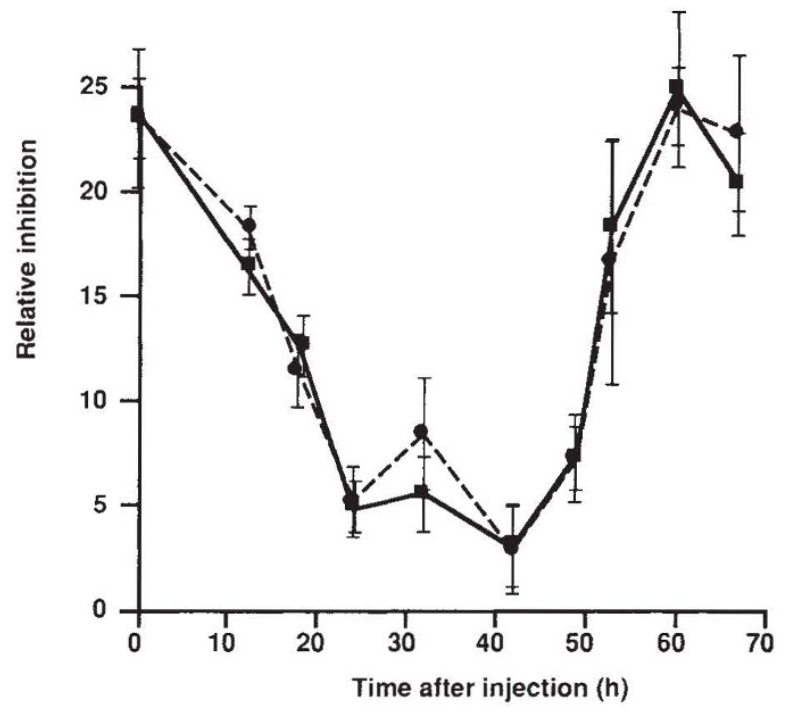

FIG. 1 Time course of $\mathrm{Ca}^{2+}$ current inhibition by somatostatin or carbachol in $\mathrm{GH}_{3}$ cells. At time zero, cells were injected with antisense oligonucleotide $\beta$-com (see Methods). At the indicated time points, the $\mathrm{Ca}^{2+}$ current was measured in the presence of $1 \mu \mathrm{M}$ somatostatin (dotted line) or $10 \mu \mathrm{M}$ carbachol (solid line). Mean values with s.e.m. are shown $(n \geqq 5)$.

METHODS. Microinjection (about 10,000 full-length oligonucleotides per nucleus) and electrophysiological measurements were done as described ${ }^{21}$, but during current recording cells were perfused with a solution containing $120 \mathrm{mM}$ choline- $\mathrm{Cl}, 10.8 \mathrm{mM} \mathrm{BaCl}, 1 \mathrm{mM} \mathrm{MgCl}, 5.4 \mathrm{mM} \mathrm{CsCl}, 10 \mathrm{mM}$ glucose and $10 \mathrm{mM}$ tetraethylammonium-HEPES (pH 7.4 at $\left.37^{\circ} \mathrm{C}\right)$. Sequence of injected $\beta$-com oligonucleotide: 5 '-TTGCAGTTGAAGTCGTCRTA-3', corresponding to nucleotides $825-844$ of the identical strand of the $\beta_{1}$ gene sequence $^{5}$. It can hybridize with the mRNAs of $\beta_{1}, \beta_{2}, \beta_{3}$ and $\beta_{4}$. Abbreviations for wobbled positions: $R$ ( $G$ or $A$ ), $M(A$ or $C), Y(T$ or $C), S(G$ or $C$ ). The oligonucleotide $\beta$-com is different from the previously used oligonucleotide anti- $\beta^{21}$. Oligonucleotide $\beta$-com can hybridize perfectly with $\beta_{1}, \beta_{2}$, and $\beta_{3}$ mRNAs, and the longest continuously matching stretch in $\beta_{4}$ mRNA is 16 bases. The much-improved hybridization characteristics compared with oligonucleotide anti- $\beta$ may explain why less $\beta$-com $(<10,000$ molecules, as compared with 50,000 for anti- $\beta$ ) is needed for effective repression of hormone responses. 